\title{
Refining the use of neoadjuvant chemotherapy in locally advanced bladder cancer: from conviction to optimization
}

\author{
Stéphane Culine ${ }^{1}$, Yves Allory ${ }^{2}$, Christian Pfister $^{3}$ \\ ${ }^{1}$ Department of Medical Oncology, Hôpital Saint-Louis, AP-HP, and Paris-Diderot University, Paris, France; ${ }^{2}$ Department of Pathology, Institut \\ Curie, Saint-Cloud, France; ${ }^{3}$ Department of Urology, Centre Hospitalier Universitaire, Rouen University, Rouen, France \\ Correspondence to: Stéphane Culine. Department of Medical Oncology, Hôpital Saint-Louis, AP-HP, and Paris-Diderot University, 1, Avenue Claude \\ Vellefaux 75010, Paris, France. Email: stephane.culine@aphp.fr. \\ Provenance: This is a Guest Editorial commissioned by Section Editor Xiao Li (Department of Urology, Jiangsu Cancer Hospital \& Jiangsu Institute \\ of Cancer Research \& Nanjing Medical University Affiliated Cancer Hospital, Nanjing, China). \\ Comment on: Zargar H, Shah JB, van Rhijn BW, et al. Neoadjuvant dose dense MVAC versus gemcitabine and cisplatin in patients with cT3-4aN0M0 \\ bladder cancer treated with radical cystectomy. J Urol 2018;199:1452-8.
}

Submitted Jun 11, 2018. Accepted for publication Jun 20, 2018.

doi: $10.21037 /$ tau.2018.06.11

View this article at: http://dx.doi.org/10.21037/tau.2018.06.11

Radical cystectomy (RC) remains the standard of care as local treatment of muscle-invasive bladder cancer (MIBC). However, the cancer-specific survival is approximately $50 \%$, depending on the presence of extravesical extension and lymph node metastases. In other words, nearly $50 \%$ of patients develop metastases within 2 years after RC, implying the presence of micro-metastases at the time of surgery. Neoadjuvant chemotherapy (NAC) has been shown to improve survival of patients and international guidelines recommend NAC based on the available level I evidence $(1,2)$.

\section{From clinical trials to daily practice: an ever- present need for conviction}

Despite randomized studies and meta-analyses demonstrating the survival benefit of cisplatin-based combination chemotherapy before RC, practice studies reported in the mid-2000s showed very low implementation of NAC (less than 10\%) in daily practice. Potential reasons include patient ineligibility related to renal dysfunction, toxicity of NAC, delay in performing RC, patient preference against chemotherapy, and probably most importantly low propensity of urologists and medical oncologists to use NAC. After many years of practice lagging behind evidence, more recent surveys suggest that the use of NAC has been increasing worldwide during the 2010s (3-6). However, it remains clear that not all patients who are likely to benefit from NAC receive it, suggesting that continuous efforts are required to convince more urologists and oncologists to use it.

\section{What is the optimal chemotherapy regimen? $\mathbf{A}$ need for clarification}

Level I evidence regarding the overall survival benefit of NAC was obtained with cisplatin-based regimens that are no longer currently used, such as the 4-week standard MVAC regimen which combines methotrexate, vinblastine, doxorubicin and cisplatin, or the 3-week CMV, which includes the same drugs without doxorubicin $(7,8)$. Since standard MVAC has been supplanted in the metastatic setting by less toxic regimens, namely gemcitabine-cisplatin (GC) and dose-dense MVAC (ddMVAC), efforts are being made to evaluate these regimens in the preoperative setting.

In the June 2018 issue of the Fournal of Urology, Zargar et al. reviewed the medical records of 319 patients with cT3-4aN0M0 MIBC who underwent NAC and RC from 2000 to 2015 in 20 institutions (9). One hundred patients received ddMVAC and 219 were treated with GC. Baseline characteristics were similar between the two groups except for age (patients who received ddMVAC were younger) and the proportion of variant histology features (higher in the ddMVAC group). Overall $90 \%$ and $86.3 \%$ of patients received 3 or 4 cycles of NAC in the ddMVAC and GC groups, respectively. A significantly lower rate 
of pathological complete response rates (ypT0N0) were observed in patients treated with GC $(14.6 \%$ vs. $28 \%$, $\mathrm{P}=0.005)$. Similarly, the pathological partial response rates (ypT1N0 or less) was lower (30.1\% vs. $41 \%, \mathrm{P}=0.07)$. These results translated in longer overall survival for patients treated with ddMVAC (mean survival of $7 v s .4 .6$ years, $\mathrm{P}=0.001$ ). In multivariate analysis, lymph node invasion, hydronephrosis and GC regimen were independently associated with shorter overall survival.

This study suggests that ddMVAC could be the optimal regimen for NAC. As ddMVAC is administered on a shortened 2-week schedule with G-CSF support as compared to classic MVAC, this amounts to doubling the dose-intensity of cisplatin and doxorubicin while reducing by one third the dose-intensity of methotrexate and vinblastine. In metastatic disease, a phase III trial comparing classic MVAC to ddMVAC showed an improvement in complete response rate from $11 \%$ to $25 \%(\mathrm{P}=0.006)$ and a significant benefit on overall survival from $13.5 \%$ to $22 \%$ at 5 years $(\mathrm{P}=0.04)$ in the ddMAVC arm (10). These findings could result from the doubling of the cisplatin dose intensity and may suggest that an increase in pathological response rate could be achieved with ddMVAC in the neoadjuvant setting. However other single institution, retrospective series reported comparable outcomes of GC and ddMVAC regimens $(11,12)$. Considering the retrospective, nonrandomized design of these studies, we must be guarded in drawing definitive conclusions for daily practice. Clarification to answer this question will be hopefully provided by the results of the recently closed VESPER study conducted by the French Genito-urinary Tumor Group (GETUG). Five hundred patients were randomized to receive chemotherapy for 3 months with either 6 cycles of ddMAVC or 4 cycles of GC in the perioperative setting. The issue of the optimal number of cycles will thus also be assessed.

\section{Which patients benefit from the NAC? The need for accurate biomarkers}

The identification of patients most likely to benefit from NAC certainly would help to a better implementation in daily practice. In recent years, progress in genomic characterization of urothelial cancers led to the identification of molecular subtypes $(13,14)$. Although further harmonization in subtype classifications published so far is required for clinical applicability, patients with basal/squamous-like tumors have been reported as best potential candidates for NAC. In the same time, DNA damage response-associated genes could be of interest in the prediction of response to cisplatin-based NAC. Data collectively suggest better outcome in tumors with deleterious DNA repair mechanisms (15). However clinical validation in prospective trials is required before using these promising approaches in the daily management of patients.

\section{At the end of the beginning}

There is still a long way to go to cure the majority of patients with bladder cancer and progress will only be possible with a multidisciplinary approach. The main objectives for the coming years will be to better select patients who may benefit from perioperative medical treatments, to evaluate the efficacy of immunotherapy and its potential role alongside chemotherapy, and to propose an alternative to chemotherapy for patients unfit for cisplatin.

\section{Acknowledgements}

None.

\section{Footnote}

Conflicts of Interest: The authors have no conflicts of interest to declare.

\section{References}

1. Milowsky MI, Rumble RB, Booth CM, et al. Guideline on muscle-invasive and metastatic bladder cancer (European Association of Urology Guideline): American Society of Clinical Oncology Clinical Practice Guideline Endorsement. J Clin Oncol 2016;34:1945-52.

2. Alfred Witjes J, Lebret T, Compérat EM, et al. Updated 2016 EAU guidelines on muscle-invasive and metastatic bladder cancer. Eur Urol 2017;71:462-75.

3. Reardon ZD, Patel SG, Zaid HB, et al. Trends in use of perioperative chemotherapy for localized and locally advanced muscle-invasive bladder cancer: a sign of changing tides. Eur Urol 2015;67:165-70.

4. Hermans TJ, Fransen van de Putte EE, Horenblas S, et al. Perioperative treatment and radical cystectomy for bladder cancer - A population-based trend analysis of 10,338 patients in the Netherlands. Eur J Cancer 2016;54:18-26.

5. Anan G, Hatakeyama S, Fujita N, et al. Trends in neoadjuvant chemotherapy use and oncological outcomes 
for muscle-invasive bladder cancer in Japan: a multicenter study. Oncotarget 2017;8:86130-42.

6. Booth CM, Karim S, Brennan K, et al. Perioperative chemotherapy for bladder cancer in the general population: are practice patterns finally changing? Urol Oncol 2018;36:89.e13-20.

7. Grossman HB, Natale RB, Tangen CM, et al. Neoadjuvant chemotherapy plus cystectomy compared with cystectomy alone for locally advanced bladder cancer. N Engl J Med 2003;349:859-66.

8. International collaboration of trialists. International phase III trial assessing neoadjuvant cisplatin, methotrexate, and vinblastine chemotherapy for muscle-invasive bladder cancer: long-term results of the BA06 30894 trial. J Clin Oncol 2011;29:2171-7.

9. Zargar H, Shah JB, van Rhijn BW, et al. Neoadjuvant dose dense MVAC versus gemcitabine and cisplatin in patients with cT3-4aN0M0 bladder cancer treated with radical cystectomy. J Urol 2018;199:1452-8.

10. Sternberg CN, de Mulder P, Schornagel JH, et al. Seven year update of an EORTC phase III trial of high-dose intensity M-VAC chemotherapy and G-CSF versus classic $\mathrm{M}-\mathrm{VAC}$ in advanced urothelial tract tumours. Eur J

Cite this article as: Culine S, Allory Y, Pfister C. Refining the use of neoadjuvant chemotherapy in locally advanced bladder cancer: from conviction to optimization. Transl Androl Urol 2018;7(4):757-759. doi: 10.21037/tau.2018.06.11
Cancer 2006;42:50-4.

11. Galsky MD, Pal SK, Chowdury S, et al. Comparative effectiveness of gemcitabine plus cisplatin versus methotrexate, vinblastine, doxorubicin, plus cisplatin as neoadjuvant therapy for muscle-invasive bladder cancer. Cancer 2015;121:2586-93.

12. van de Putte EE, Mertens LS, Meijer RP, et al. Neoadjuvant induction dose-dense MVAC for muscle invasive bladder cancer: efficacy and safety compared with classic MVAC and gemcitabine/cisplatin. World J Urol 2016;34:157-62.

13. Lerner SP, McConkey DJ, Hoadley KA, et al. Bladder cancer molecular taxonomy: summary from a consensus meeting. Bladder Cancer 2016;2:37-47.

14. Seiler R, Ashab HA, Erho N, et al. Impact of molecular subtypes in muscle-invasive bladder cancer on predicting response and survival after neoadjuvant chemotherapy. Eur Urol 2017;72:544-54.

15. Wezel F, Vallo S, Roghmann F, et al. Do we have biomarkers to predict response to neoadjuvant and adjuvant chemotherapy and immunotherapy in bladder cancer? Transl Androl Urol 2017;6:1067-80. 